\title{
Chapter 1 of the Book Titled: "Telecommunication Network Performance and Quality of Service": The Concept of Telecommunication Network Performance and Quality of Service
}

\author{
Sigit Haryadi \\ Institut Teknologi Bandung
}

DOI: $10.17605 / O S F . I O / C Q 8 N A$

Citation: Haryadi, S. (2018, January 25). Chapter 1. The Concept of Telecommunication Network Performance and Quality of Service. Retrieved from osf.io/mukqb

\subsection{General Concept of NP and QoS}

ITU-T Rec. E.800 provides a set of commonly used terms in the study and management of Network Performance (NP) and Quality of Service (QoS). In detail, the technical and non-technical terms related to the QoS listed and intended to represent the interests of all parties of telecommunications service market, i.e., user, service provider, manufacturer and regulator.

Based on ITU-T Recommendation E.800 [5], quality \& performance in the telecommunications field can be divided into several levels as follows:

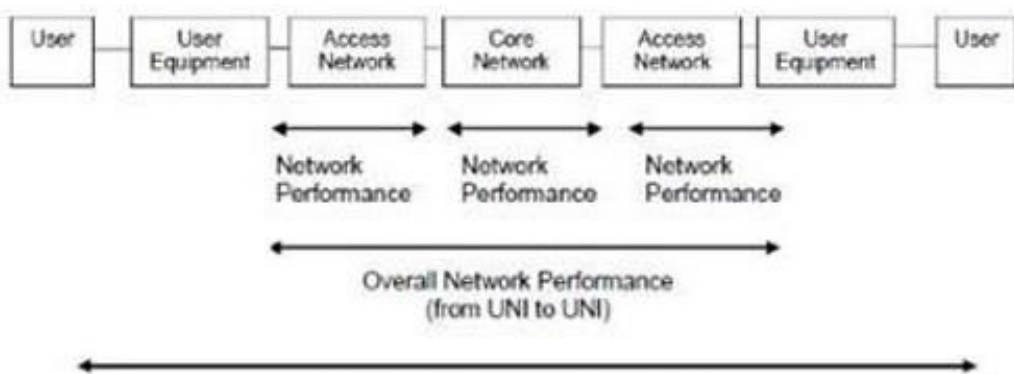

End-to-end Quality of Service (QoS)

Quality of Expenenco (QOE)

Fig.1. Classification of NP, QoS and QoE according to ITU-T 
Note: In the era of OTT services (Over-the-top services), the servers built by OTT providers are positioning as if replacing Core-networks of networks built by Telco.

Here's an explanation of the classification:

[1]NP (Network Performance): the performance of a network element. Typically, the NP is determined by assessing several technical parameters that describe the performance of a network element with reference to some particular standard. NP parameter Example: BER (Bit Error Ratio), Transmission Delay, processing time, jitter.

[2] Overall Network Performance: The performance of all elements is integrated to form a network so that the network can be considered as a "black box" that will measure performance. For example, performance measurements between two UNI (User Network Interfaces).

[3]End-to-end QoS: overall performance of the elements and processes involved in the chain of transmission of a telecommunications service until the service performed. QoS is measured from the point of view of the user but does not involve the user in the process of measurement and assessment carried out objectively. Typically, QoS measurements depend on some typical characteristics of a telecommunications service.

[4]QoE (Quality of Experience): overall performance of a telecommunications service by involving users in the process of measurement. QoE assessment is more subjective because it is influenced by the experience and the level of expectations of users of a telecommunications service. 


\subsubsection{Definition of the QoS (Quality of Service)}

Quality of Service (QoS) consists of a set of parameters related to the performance of traffic on telecommunication network. QoS definitions contained in the ITU-T as:

\section{"The collective effect of service performance which determine the degree of service user satisfaction" [5].}

In addition to the above definition, there are several other QoS concepts:

i. Service support performance

ii. Service operability performance

iii. Severability performance and

iv. Service security performance

Complete definition contained in the ITU-T E.800 series. Telecommunication operators and service providers are able to provide better QoS, are more likely to retain existing customers and may increase again from rival operator customer churn. But a better QoS requires greater costs. Normally, each operator will balance QoS and cost.

\subsubsection{Grade of Service Definition}

People who are the first time in learning the telecommunications system, often confused with the difference in terms of quality of service (QoS) and service 1eve1 (GOS).

GOS definitions contained in other ITU-T, the E.600 series. Based on ITU-T, the definition of COS is: 


\section{A number of traffic engineering variables to provide a measure of adequacy of a group of resources under specified conditions (ITU-T E.600)}

\subsubsection{GOS of Circuit-Switched Network}

The most important Grade of Service parameter of the Circuit Switched Network is the blocking probability.

Blocking probability is the most conventional COS, and occurs in the loss network of the telephony service, where there is no buffer in the system as a place to wait for the traffic congestion. Blocking probability is the value of loss call calculated in the form of cal1congestion, and the notation is B, refer to Erlang-B Formula. In the network, which has the Poisson distribution traffic, 1oss-cal1 is calculated using the Erlang-B formula. In the network, which has the Gaussian distribution traffic, 1oss-cal1 is calculated using the norma1-1oss formula. 


\section{End to End Blocking Probability.}

The calculation of end-to-end blocking probability (NNGOS) is used as a basis for planning of end-to-end network performance. For example, assume that the blocking probability of each route $=p_{i}$; the carried probability of each route $=q_{i}=\left(1-p_{i}\right)$ and the end-to-end blocking probability $=p_{\text {ete }}$.

i. The end-to-end blocking probability formula for the serial Tinks:

$$
p_{\text {end to end of serial nodes }}=1-\prod_{i=1}^{N}\left(1-p_{i}\right)
$$

Where pi = blocking probability of each node

ii. The end-to-end blocking probability formula for the para1e1 1inks:

$$
p_{\text {end-to-end of parallel nodes }}=\prod_{i=1}^{N} p_{i}
$$

Problem 1: Refer to the following network topology

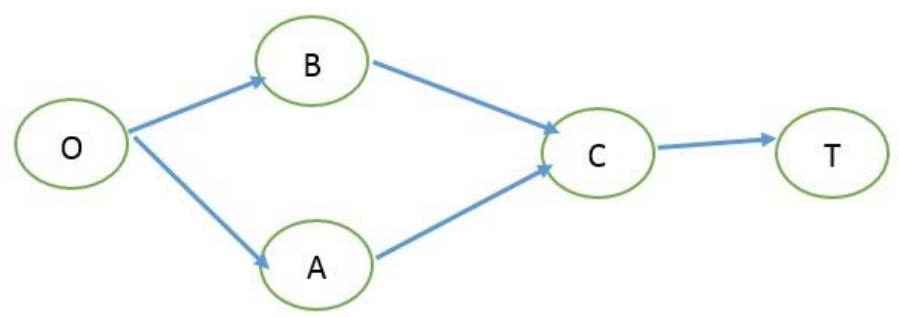

Data:

\begin{tabular}{|c|c|c|}
\hline Route & Total Delay (= propagation delay + server delay) in ms & Probability of Blocking in 0.001 \\
\hline OA & 100 & 5 \\
\hline OB & 110 & 8 \\
\hline BC & 120 & 10 \\
\hline AC & 130 & 15 \\
\hline CT & 50 & 20 \\
\hline
\end{tabular}

Calculate: (1) End-to-end Blocking probability of route OBC; (2) End-to-end Blocking probability of route OAC; (3) End-to-end Blocking probability of route OT; and end-to-end delay OT. 


\section{Solution:}

(1) End-to-end Blocking probability of route OBC (= rute OB serial BC) $=1-(1-0.008) *(1-$ $0.010)=0.01792$

(2) End-to-end Blocking probability of route OAC (= rute OA serial AC) $=1-(1-0.005) *(1-$ $0.015)=0.019925$

(3) End-to-end Blocking probability of route OT (= (route OBC parallel r0ute OAC) serial route CT $)=1-(1-(0.01792 * 0.019925)) *(1-0.02)=0.0203499149$

(4) end-to-end delay OT: (a) OBCT delay $=110+120+50=280 \mathrm{~ms}$, and (b) OACT delay $=100+130+50=280 \mathrm{~ms}$

\subsubsection{GOS of the Internet Protocol Network}

Refer to ITU-T G.1010 [3], the three most important COS parameters for the Internet traffic is latency, jitter and packet 1oss.

1) Latency .

End-to-end delay computation is done by adding up the overall delay experienced during the network elements = total of the packet transfer delay or processing time + total of the propagation delay.

\section{a. Packet Transfer Delay}

Packet transfer delay = Sojourn Time (Waiting time in buffer + processing time in the server) is a term to indicate the time required for the packet in the system = the time in the buffer + the time in the server.

\section{b. Propagation Delay.}

Propagation delay is the time taken by a packet while transmitted in the transmission medium. Wi11 be even greater if the transmission distance is farther away. For radio communications, the wave velocity approaches the speed of 1ight, then the propagation delay experienced by packets is about 3.33 microseconds at each transmission distance of $1 \mathrm{~km}$. In the communication cables at the speed of light around 0.6 to 0.8 of the speed of light, the 
propagation delay experienced by a packet is about 4.5 microseconds per transmission distance of $1 \mathrm{~km}$.

2) Jitter

Jitter is an important end-to-end COS parameters. There are two terms of jitter, the time jitter and frequency jittter.

\section{a. Time Jitter.}

Time Jitter in the telecommunication system is undesirable deviations from end-to-end delay. Time jitter occurs due to end-to-end delay varies with time. To calculate the time-jitter, first create end-to-end delay curves as a function of time, then look for when there is a maximum or minimum peak value, and then calculate the period between the two peak values, and time jitter is twice of this period. Time jitter to cause deterioration in the quality of real-time connection, such as VoIP, The talk sounded intermittent.

\section{b. Frequency Jitter}

Frequency Jittter a scale of frequency, frequency jitter is the inverse of the time jitter. Frequency Jitter can be caused by electromagnetic interference and crosstalk with other signal carriers. Frequency Jitter can affect the performance of processors and network elements, and can also cause packet loss.

3) Packet Loss.

Packet loss calculation is very complicated, especially if the capacity of the buffer size is not infinite. If the infinite buffer capacity, theoretically there would be no packet loss. Packet loss calculation should take into account the packet arrival distribution, the distribution of the service on the 
server, the number of servers, buffer capacity and queuing disciplines. In this book does not explain the method of calculation of packet 1oss.

Problem 2: Refer to Problem 1, Calculate: (1) End-to-end delay if the route is OBCT; (2) End-to-end delay if the route is OACT

\title{
1.1.2.3. The ITU-T Packet Switched GOS Parameter
}

In the ITU-T recommendation E.726 series equivalent to E.721, there COS parameters for packet-switched networks, GOS standards for cal1 level is not the same as the standard for packet-1evel GOS. Some parameters for packet level GOS includes:

\author{
i. Packet transfer delay \\ ii. Packet delay variation \\ iii. Severe7y error packet block ratio \\ iv. Packet 7 oss ratio \\ v. Frame transmission delay \\ vi. Frame discard ratio
}

\subsection{QoS Models}

In the ITU-T Recommendation G.1000 [6], described the QoS model viewed from four different angles.

At a more detailed level, QoS can be divided into four viewpoints as illustrated in Figure 4. This concept is described further in [ITU-T G.1000]. A generic definition of QoS is derived from a definition of quality and is given in clause 2. Of particular interest is QoS experienced by the user (expressed by QoSE or QoSP = QoS perceived). QoSE is influenced by the delivered QoS and the psychological factors influencing the perception of 
the user. Understanding of the QoSE is of primary importance to help optimize revenue and resources of the service provider.

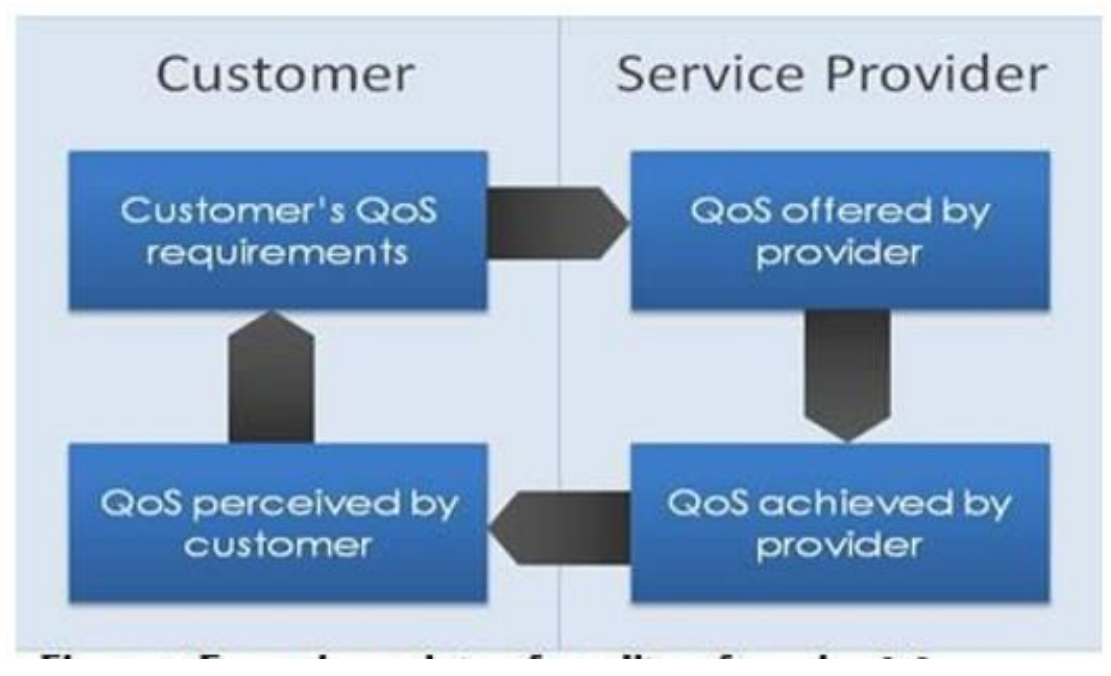

Fig. 2. Four Viewpoints of QoS [6]

Four different angles of QoS Model according to the ITU-T can be described as follows:

i. QoSR (QoS Required by the User). QoS needs of customers are usually described in non-technical parameters. A customer would expect the performance of a telecommunication service without considering the technical aspects or limitations in the implementation of service.

\section{ii. QoSO (QoS Offered by the Service Provider).}

Telecommunications service provider mentioned QoS levels to be achieved. This can be done in two ways:

- Non-technically, to allow users to understand the specifications given.

- Technically, in order to facilitate further discussion with experts, the determination of the SLA, and facilitate the planning of a technical nature. 
iii. QoSD (QoS Delivered by the Service Provider). QoSD reflect the level of QoS that has been successfully achieved by the telecom service providers. QoSD can test the ability of a telecommunications service provider to deliver the promised QoS.

iv. QoSE (QoS Experienced by the User). QoS experienced by users reflect the subjective point of view of a user in certain circumstances they experienced. Customer satisfaction is one of the driving factors for this type of QoS. In general, QoSE described in nontechnical parameters. Telecom service providers can measure the level of QoSE by conducting a survey to its customers or to seek advice and input from them. At this stage, a user combines personal experience with the expected technical quality of the service it uses. In addition to technological aspects, there are several other factors that affect the level of QoSE. Some of these factors such as starting from the signing of the contract between the user and the service provider, the service provider the ability to handle problem faced by customers, and the overall relationship between the customer and the service provider. Thus, it can be concluded that QoSE quite difficult to measure because there are several factors "hidden" are not easy to identify.

\subsection{Practical User Perception of QoS vs Operational Performance [1]}

Why are any differences between the results of measurements of QoSE (QoS Experience by the user) and QoSD (QoS Delivered by the provider), whereas the measurement of QoS and network performance are not contradictory? 


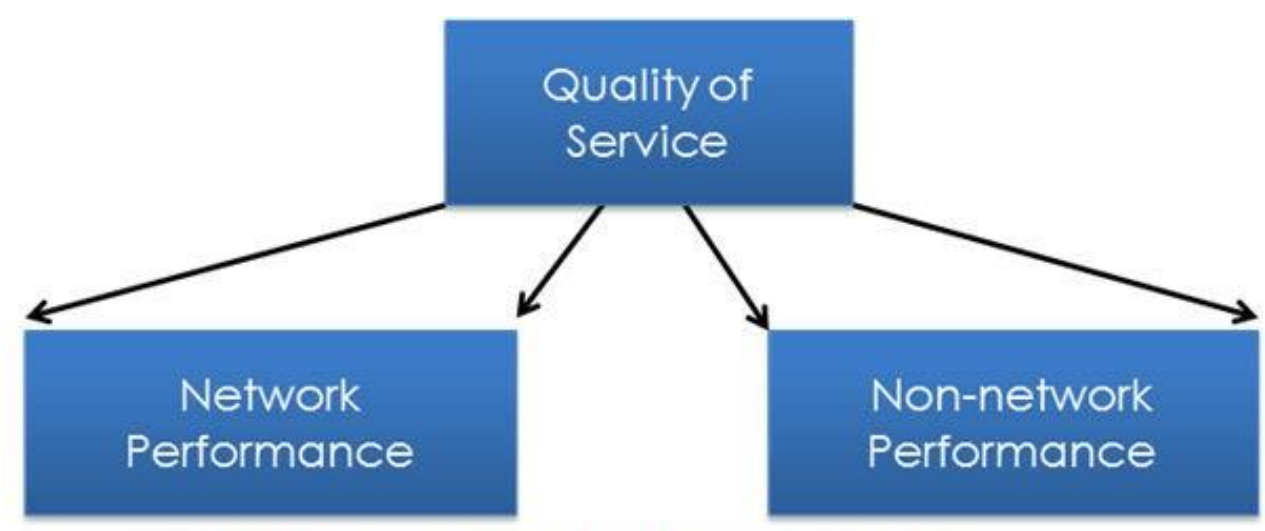

Figure 3 Quality of service comprises network and non-network criteria [6]

In practice, many factors that influence the customer's perception of the QoS service they received from the provider.

In general, the perception of the customer is to compare the quality of service that they feel with the quality they expect. Customer expectations are influenced by the rates they pay and the information that they know from the media and from books. In general, if a customer feels an expensive, then their expectations for service quality is high as well.

Provider of telecommunications equipment owned or rented, and operates with the standard of performance they called KPI (Key Performance Indicator).

The better prepared KPI, and the more realistic service rates, the correlation between customer expectations for QoS performance telecommunications systeM, will increase.

To better understand the expectations of its customers, the provider must have good customer service. Customer service should be a very good understanding of operational performance measured through Key 
Performance Indicators, as well as understand the relationship between customer complaints with performance indicators.

The task is customer service is two-way. On the one hand, they should be able to answer customer complaints properly, according to the technical conditions of operation. On the other hand, they should be able to give direction to the company, the translation of the customer's wishes into technical performance criteria.

Providers that are less, in general, ignore the customer service. As a result, customers will be frustrated. Customers have been disappointed, because he felt the complaint was not answered correctly. Provider engineers also depressed, because it was already successfully operate the device in accordance with technical standards, but it is still considered bad by the company who read so many reports of customer disappointment.

\subsection{Mobile Circuit Switched Grade of Service}

ITU-T Recommendation E.771 proposes network Grade of Service (GOS) parameters for current and evolving land mobile services. These parameters are defined, and their target values specified, assuming that the network and the network components are operating in their normal mode (i.e. are fully operational). Further, the parameters and their target values assume normal (as opposed to distress or emergency) traffic.

In this Recommendation, the following traffic GOS parameters are specified for mobile circuit switched services:

i. Post Selection Delay: defined as the time interval from the instant the first bit of the initial SETUP message containing all the selection 
digits is passed by the calling terminal to the access Signaling system until the last bit of the first message indicating ccall disposition is received by the calling terminal (ALERTING message in case of successful call).

ii. Answer signal delay: defined as the time interval from the instant that the called terminal passes the first bit of the CONNECT message to its access Signaling system until the last bit of the CONNECT message is received by the calling terminal.

iii. Call release delay: defined as the time interval from the instant the DISCONNECT message is passed by the user terminal which terminated the call to the access Signaling system, until the RELEASE message is received by the same terminal (indicating that the terminals can initiate/receive a new call).

iv. Probability of end-to-end blocking: defined as the probability that any call attempt will be unsuccessful due to a lack of network resources.

v. Probability of unsuccessful land cellular handover: defined as the probability that a handover attempt fails because of lack of radio resources in the target cell, or because of a lack of free resources for establishing the new network connection. The failure condition is based either on a specified time interval since the handover request was first issued or on a threshold on signal strength. 


\subsubsection{The General Concept of QoS by ETSI [2]}

ETSI standard TS 102 250-2 covering the QoS aspects for popular services in GSM and 3G networks. The standard divided into 6 parts book that identified below:

- ETSI TS 102250 Part 1 identifies QoS criteria for popular services in GSM and 3G networks. They are considered to be suitable for the quantitative characterization of the dominant technical QoS aspects as experienced from the customer perspective.

- ETSI TS 102250 Part 2 defines QoS parameters and their computation for popular services in GSM and 3G networks.

- ETSI TS 102250 Part 3 describes typical procedures used for QoS measurements over GSM, along with settings and parameters for such measurements.

- ETSI TS 102250 Part 4 defines the minimum requirements of QoS measurement equipment for GSM and 3G

- ETSI TS 102250 Part 5 specifies test profiles which are required to enable benchmarking of different GSM or 3G networks both within and outside national boundaries.

- ETSI TS 102250 Part 6 describes procedures to be used for statistical calculations in the field of QoS measurement of GSM and 3G networks using probing systems.

General Consideration: ETSI identifies QoS criteria for popular services in GSM and 3G. They are considered to be suitable for the quantitative characterization of the dominant technical QoS aspects as experienced from the customer perspective. The criteria are described by their name and a 
short description from the customer point of view. The relationship between customer satisfaction, QoS and NP is shown in figure 1.

\subsubsection{ETSI QoS Aspects}

Figure below shows different phases (Quality of Service aspects) during service use from the customer's point of view.

\section{Network \\ Availability \\ Network \\ Service \\ Service \\ Senice \\ Accessibility \\ Accessibility \\ Retainability \\ Integrity}

Fig. 4. ETSI's Customer viewpoint of QoS and QoE [2]

The five QoS aspects are:

i. Network Availability: is the probability of a telecommunications service that can be offered to customers through a network infrastructure.

ii. Network Accessibility: probability that users can register on the network to be successful so that the network can provide telecommunication services. Network can only be accessed when it is available to the user.

iii. Service Accessibility: probability that the user can access the service you want to use., If the customer wants to use a service, the network operator should provide him as fast as possible access to the service

iv. Service Integrity: describes QoS while using the service, and contains elements such as the quality of the content being transmitted, such as sound quality, video quality, and the number of 
bits transmitted error in the file. Service integrity can only be calculated if the service is accessible to success.

v. Service Retainability: Service retainability describes the termination of services, in accordance with or against the will of the user. Explains how to end or terminate a service, whether or not the will of the user. Examples of service retain ability parameter are call cut-off ratio or the data cut-off ratio.

\section{Customer expectation of QoS}

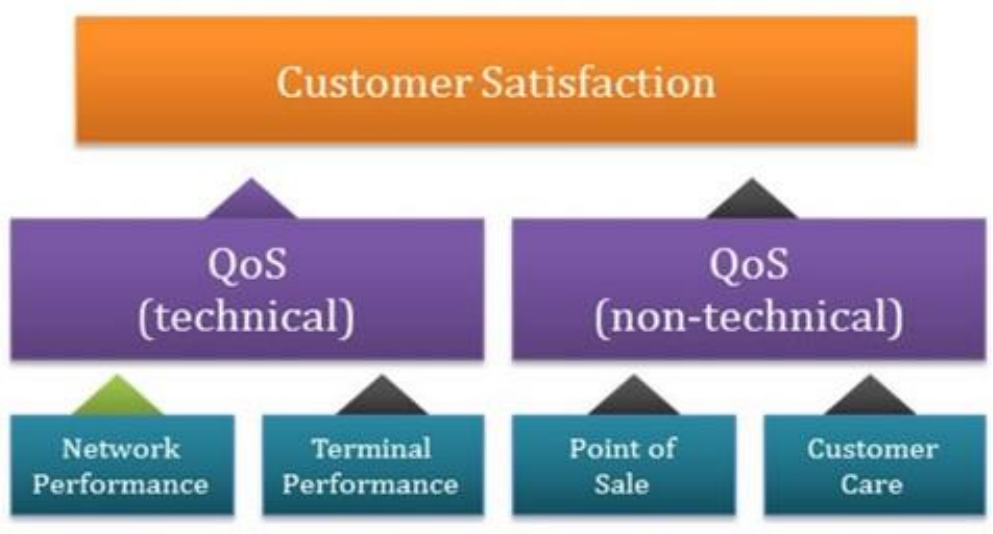

Fig.5. Relationship between Customer Satisfaction and QoS of the Provider [2]

\subsubsection{GPP Concept of QoS [7]}

The book of 3GPP Standard TS 23.207 provides the framework for endtoend GPRS and UMTS. The end-to-end QoS architecture is provided in Figure 7. The book describes the interaction between the TE/MT (Terminal Equipment/Mobile Terminal) Local Bearer Service, the GPRS Bearer Service, and the External Bearer Service, and how these together provide Quality of Service for the End-to-End Service. The book also describes IP level mechanisms necessary in providing end-to-end Quality of Service and possible interaction between the IP level and the GPRS level, as well as the application level and the IP level. 
This book covers different architectural aspects of the end-to-end Quality of Service concept and architecture with varying level of detail. In general, other specifications shall be referred to for further details; these other specifications enable the reader to acquire the full understanding of the end-to-end Quality of Service concept and architecture.

i. QoS Management Functions in the Network: to provide IP QoS endto-end, it is necessary to manage the QoS within each domain. An IP BS (Base Station) Manager is used to control the external IP bearer service. Due to the different techniques used within the IP network, this communicates to the UMTS BS manager through the Translation function. The QoS management functions for controlling the external IP bearer services and how they relate to the UMTS bearer service QoS management functions are shown in Figure

ii. QoS Conceptual Model: there are many different end-to-end scenarios that may occur from an UE connected to an UTMS network. The following examples depict how end-to-end QoS will be delivered for a number of scenarios that are considered to be significant. 


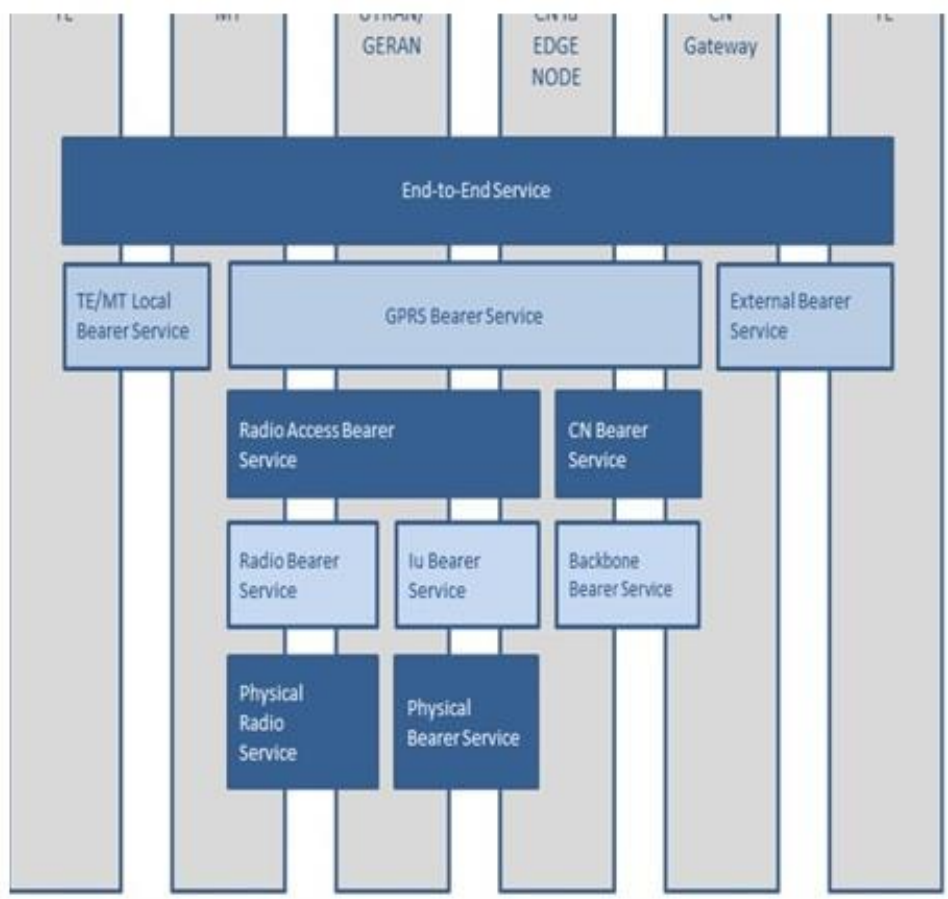

Fig. 6. End-to-end QoS of 3GPP's Standard [7]

\subsubsection{GSM Association QoS Theory [4]}

GSM Association standard PRD IR.42, defines QoS parameters and their computation. The harmonized definitions given in this book are considered as the prerequisites for comparison of QoS measurements and measurement results.

General Consideration: all the defined quality of service parameters and their computations are based on field measurements. That indicates that the measurements were made from customers point of view (full End-toEnd perspective, taking into account the needs of testing).

It is assumed that the end customer can handle his mobile and the services he wants to use (operability is not evaluated at this time). For the purpose of measurement it is assumed:

- that the service is available and not barred for any reason

- $\quad$ routing is defined correctly without errors and 
- The target subscriber equipment is ready to answer the call.

Voice quality values measured should only be employed by calls ended successfully for statistical analysis. However, measured values from calls ended unsuccessfully (e.g. dropped) should be available for additional evaluations and therefore, must be stored.

\section{Quality of Service Parameter Definition, and Their Computationof GSM Association} The following Figure 7 shows a model for quality of service parameters.

This model has three layers. The first layer is the Network Access, the basic requirement for all the other QoS aspects and QoS parameters. The outcome of this layer is the QoS parameter Network Accessibility.

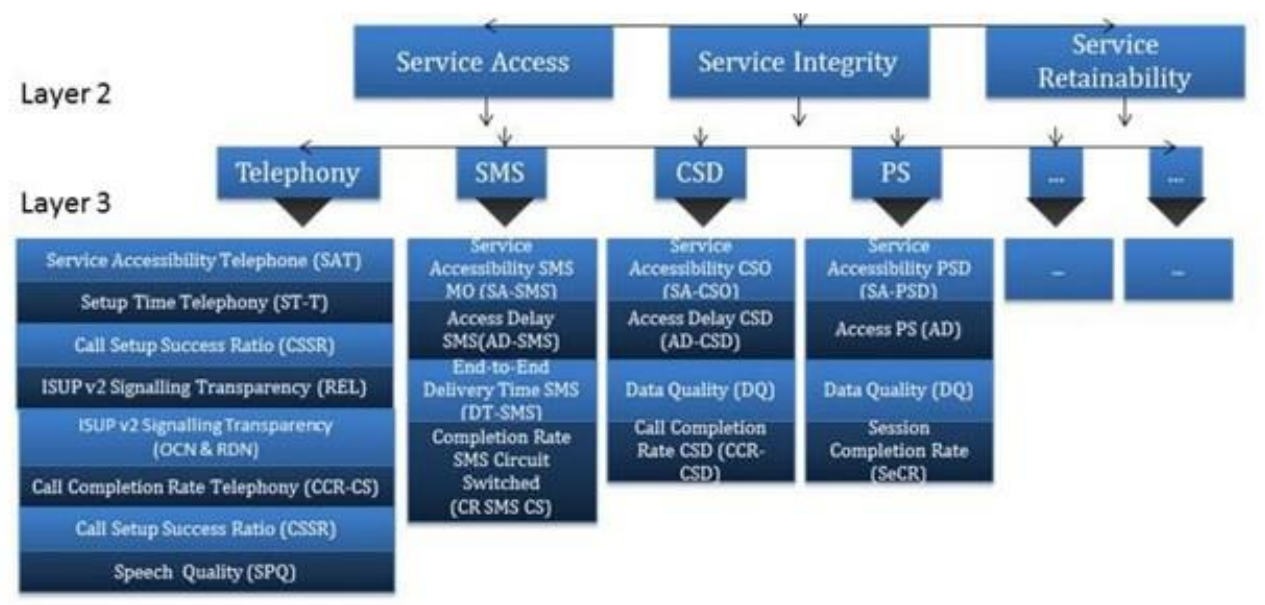

Fig. 7. QoS of GSMA

The second layer contains the other three QoS aspects Service Access, Service Integrity and Service Retainability. The different services are located in the third layer. Their outcome is the QoS parameters. 


\subsection{Telecommunication Network Topology}

a. PSTN Topology

PSTN (Public Switched Telephone Network Topology)

Phone (Fix Customer Premise Equipment)

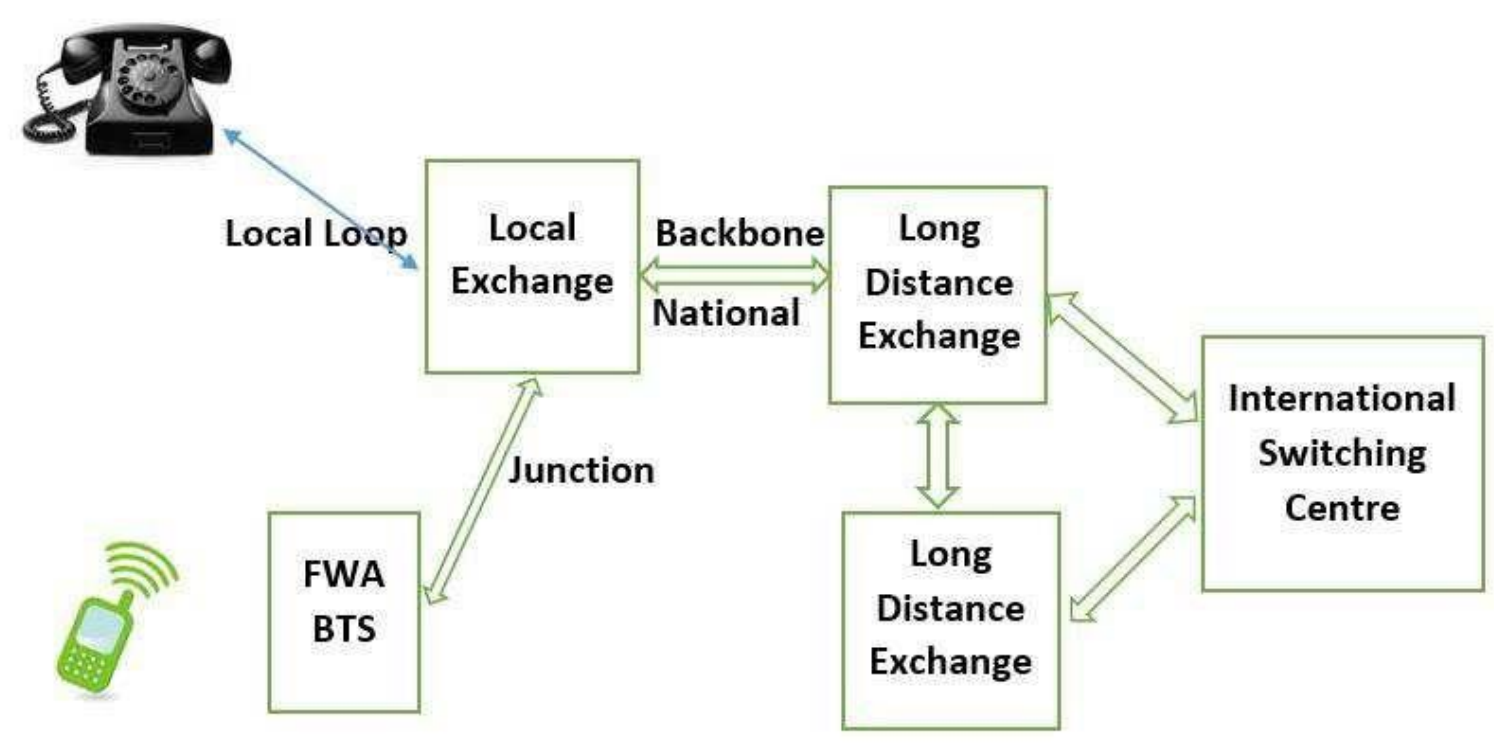

Radio Phone (Fix Wireless Access phone)

b. FTTH network topology, source:

http://www.fiberoptictel.com/application-solution/basic-

fttxnetworks/page/3/

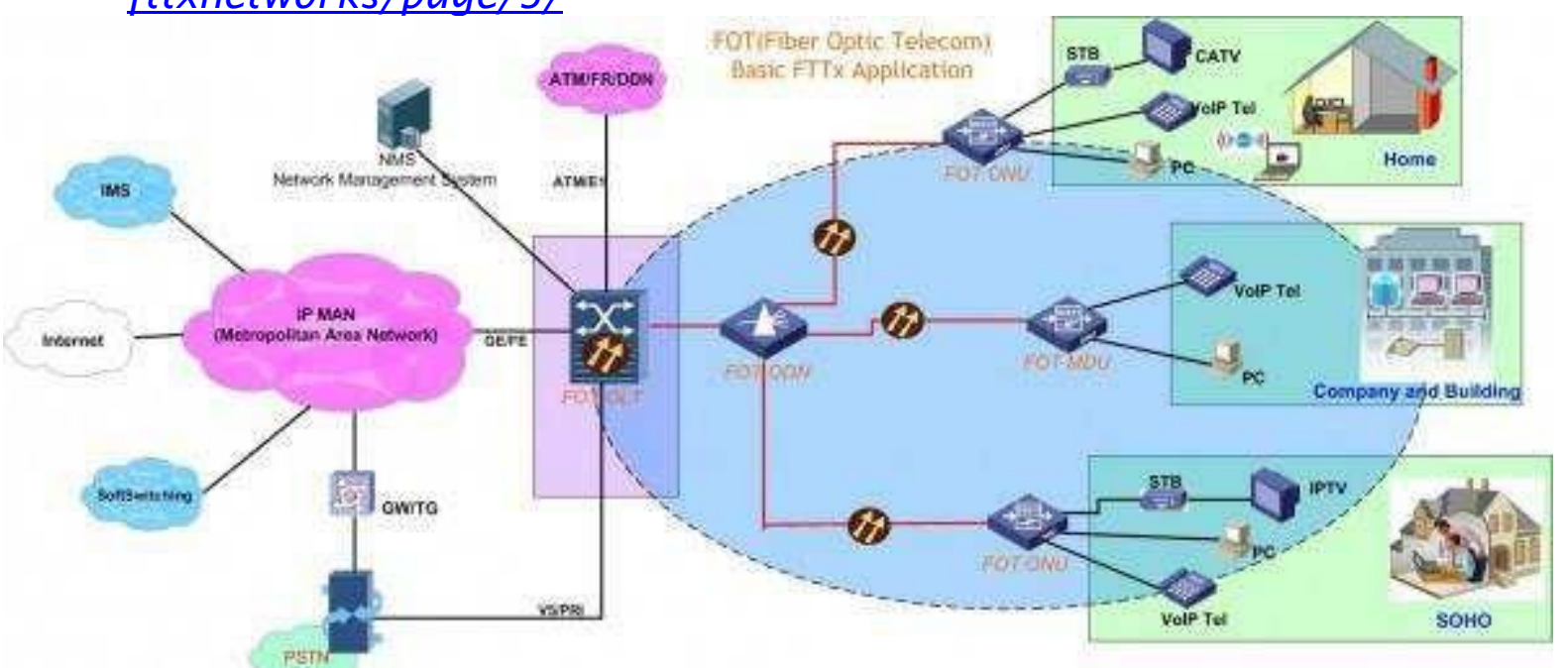


c. 2G network topology

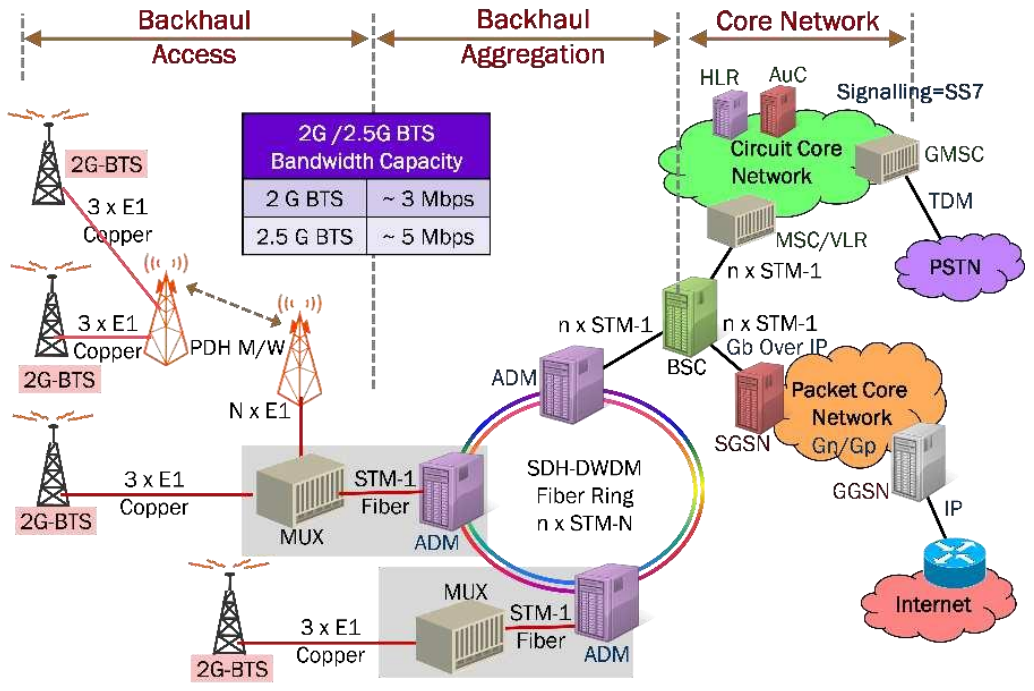

d. 2G-GSM User/data plane topology, Source: http://ipcisco.com/evolution-from-2g-to-Ite-part-1/

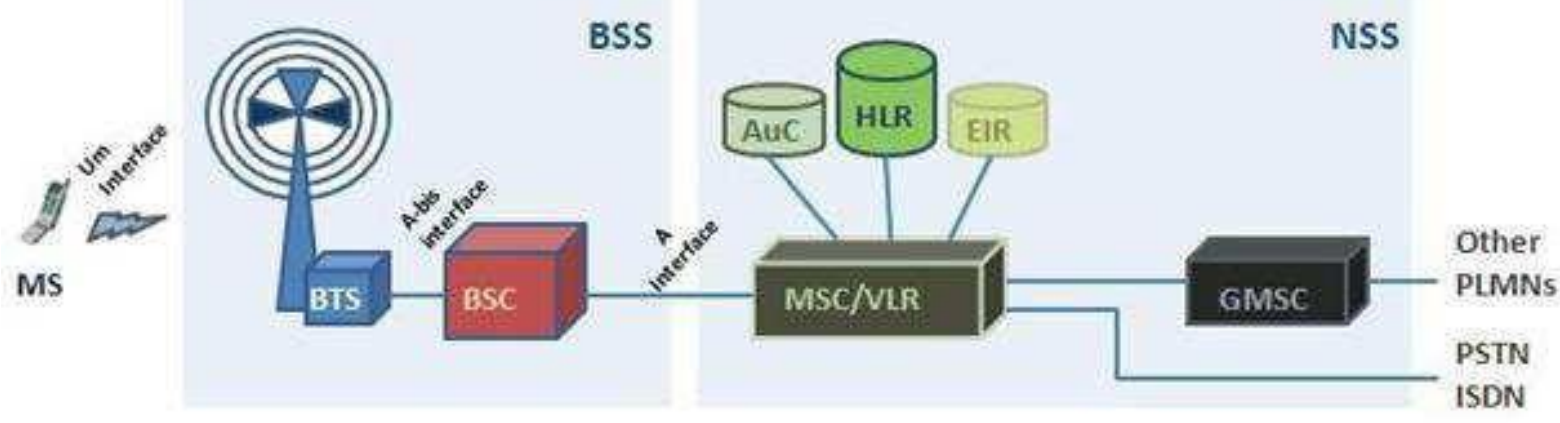

e. 2G- GPRS User/data plane topology, Source: http://ipcisco.com/evolution-from-2g-to-Ite-part-2/

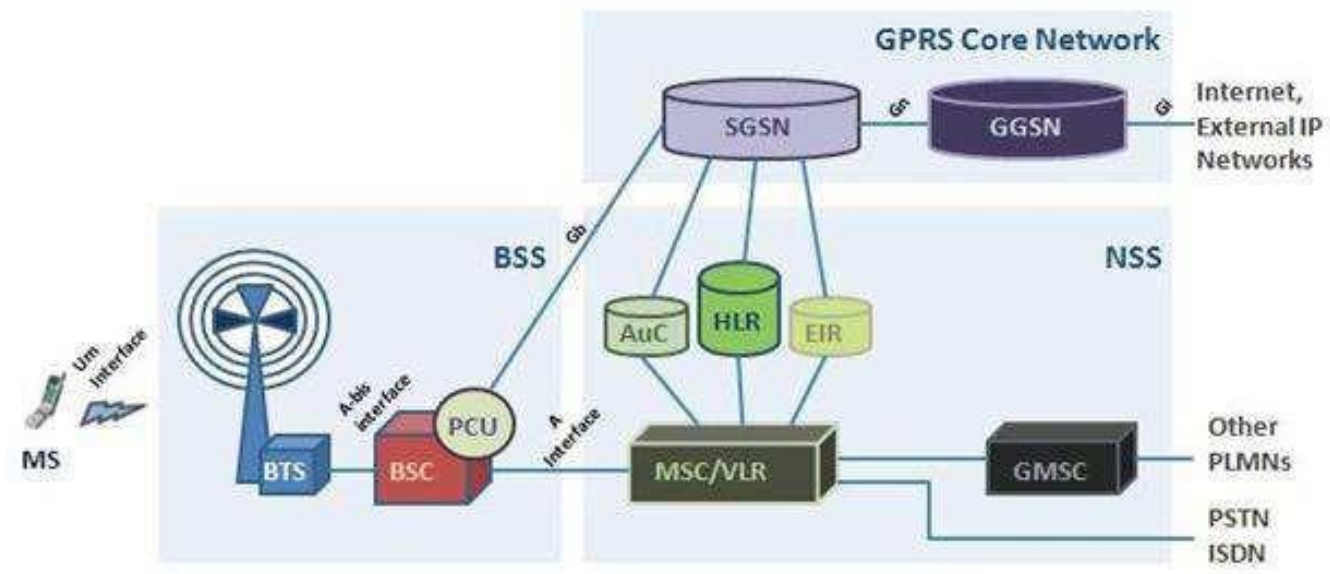


f. 3G user/data plane topology, source: http://denmasbroto.com/article-2-umts3gnetwork-architecture.html

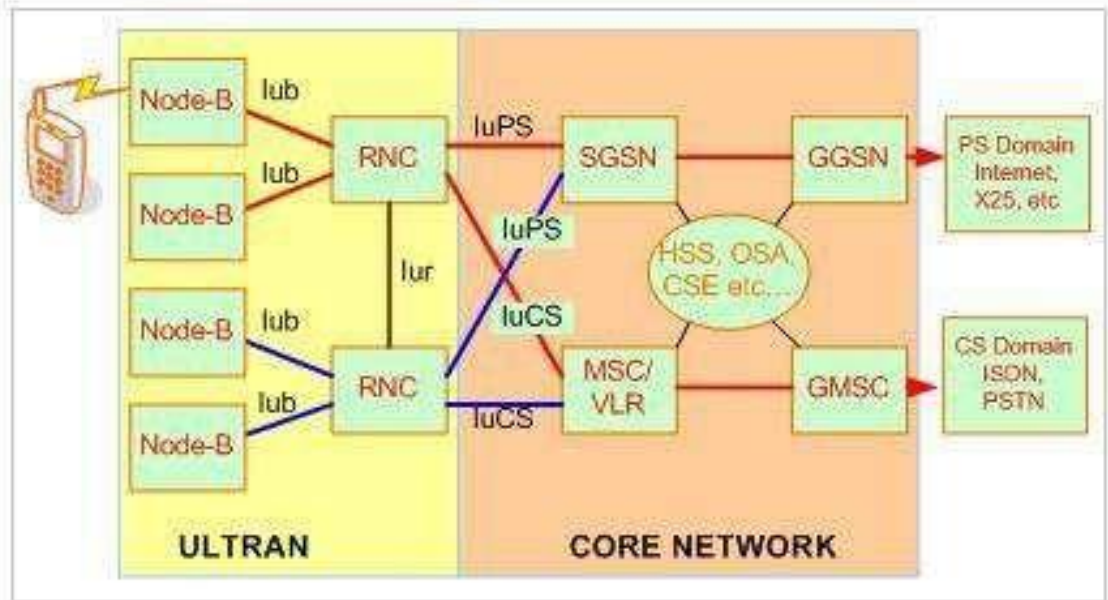

g. LTE network topology, source: http://www.rcrwireless.com/20140513/networkinfrastructure/lte/lte-networkarchitecture-diagram

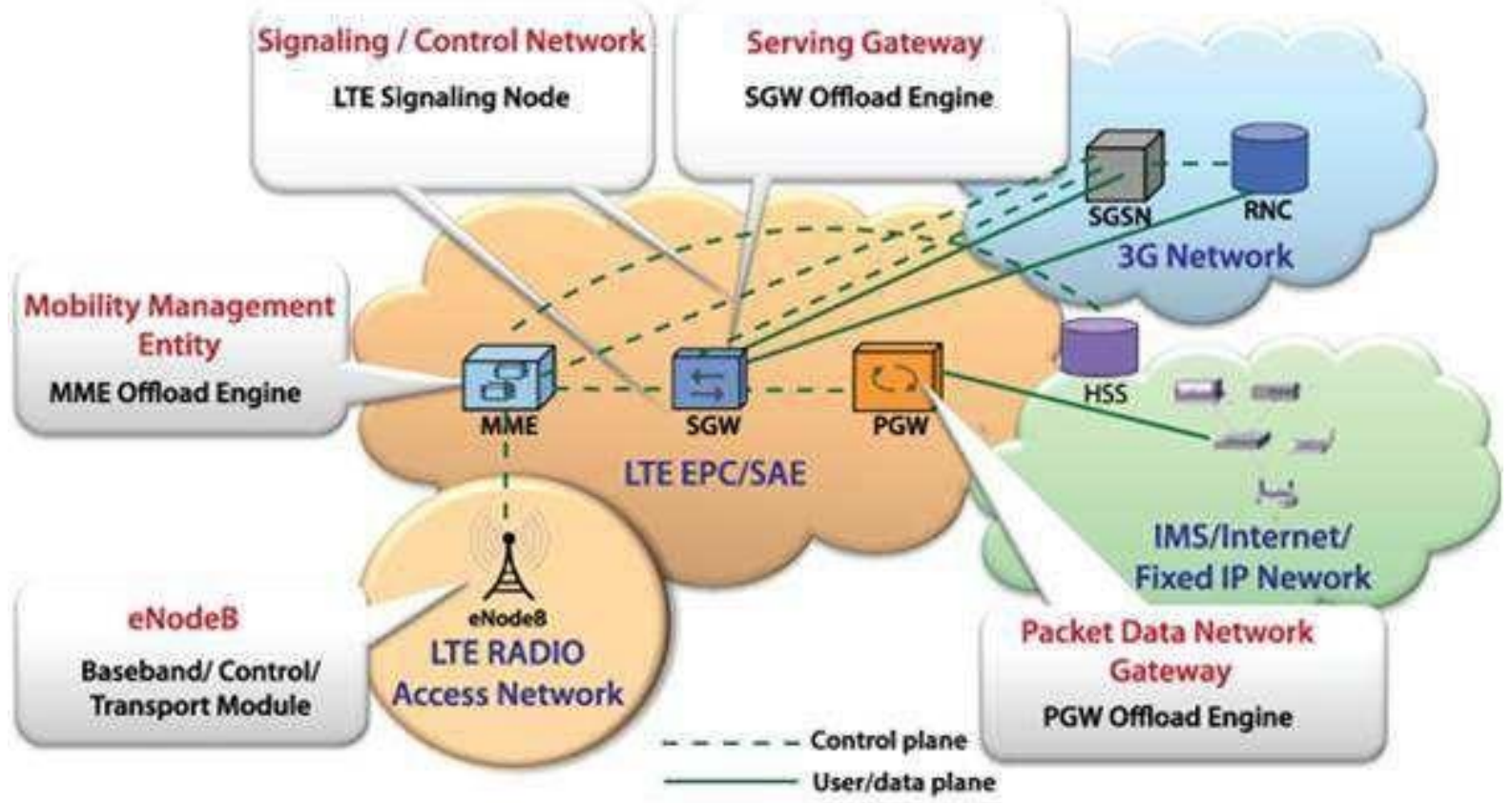




\section{h. 2G/3G user/data plane topology}

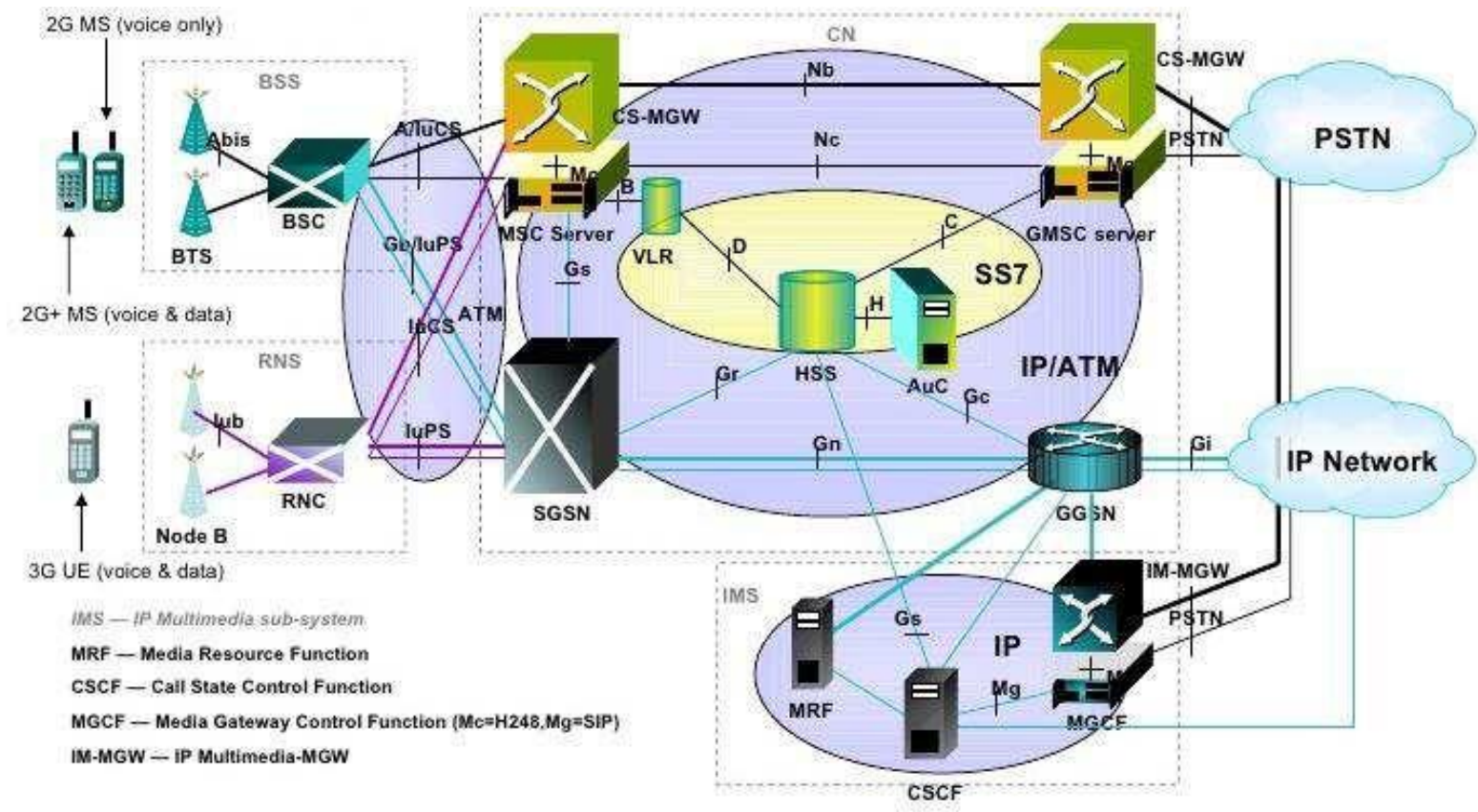

\section{i. 2G/3G/4G control plane topology}

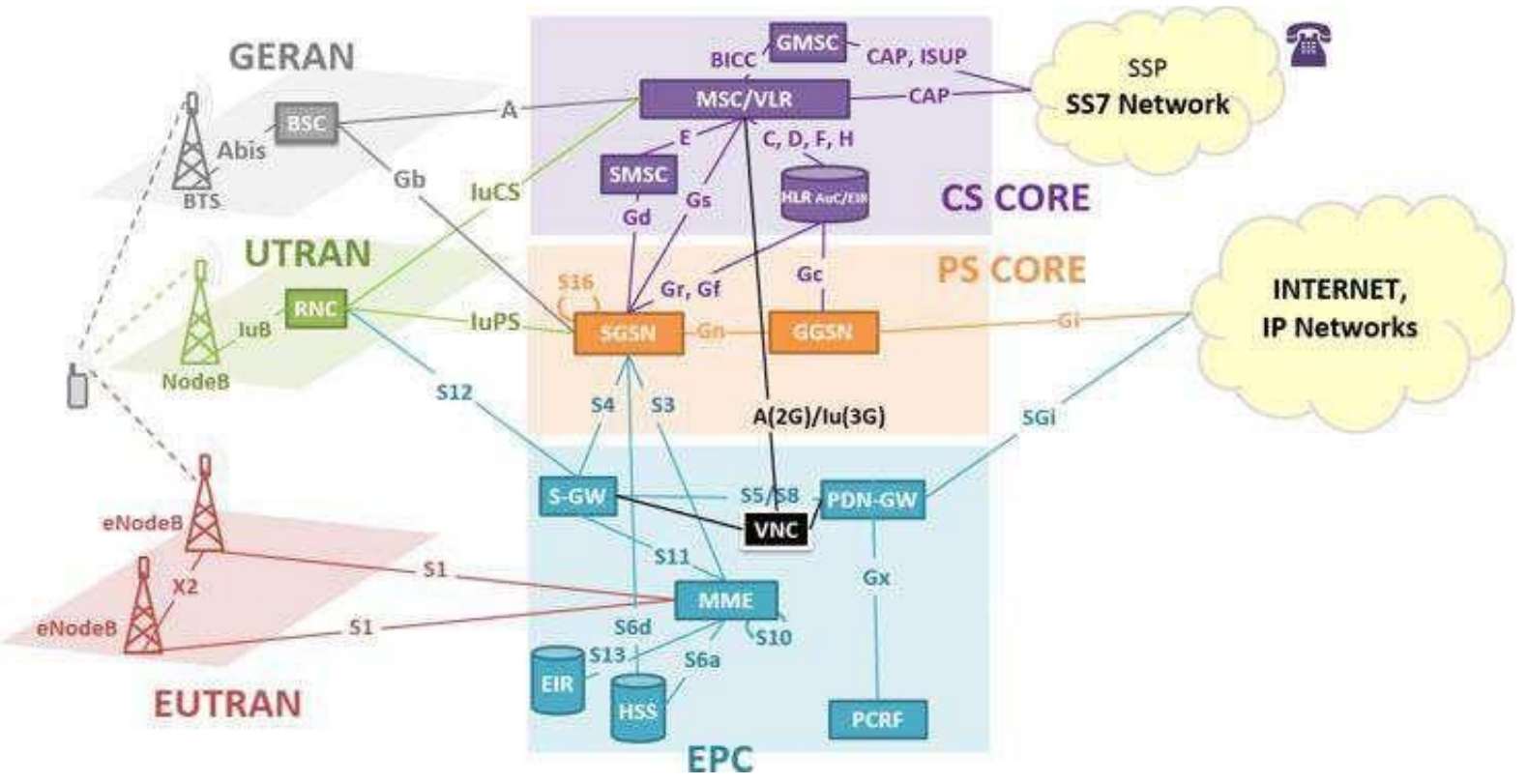

\subsection{Types of telecommunications services}

Considered in terms of the traffic distribution, the difference is the type of service. 
Many ways to classify the types of telecommunications services.

For example, here are 3 ways to group, which is based on the ITU-T, ETSI and GSMA.

\subsubsection{Grouping telecommunications services according to ETSI standards} TS.102.250 series:

i. Direct services. That includes direct services are: Telephony, HTTP (web browsing), Streaming, FTP, Ping, mobile broadcast, Push to talk and Video telephony.

ii. Store and forward service. That includes store and forward services are: E-Mai 1, SMS (Short Message Service) and MMS (Mu7timedia Message Service).

1.6.2. In the ITU-T G.1010 series, telecom services are grouped into:

i Audio services. That includes audio services are: Conversation voice, Voice messaging and High quality streaming audio;

ii Video services. That includes video services are: Videophone and One-way video

iii Data services. That includes data services are: Web browsing HTML, Bu7k data transfer / retrieval and Transaction service-high quality for a variety of purposes, such as ATM, e-commerce, command/control services, Interactive games, Telnet, E-mai7 server access, E-mail server to server transfer, real time fax, store \& forward fax, Low priority transaction and UseNet. 


\title{
1.6.3. According to the GSMA standardization PRD.IR.42 series.
}

Telecommunication services consisting of: Telephony, SMS, Circuit Switched Data Service, Packet Switched Data Service and Conversationa7 data C7ass

\begin{abstract}
Author: Sigit Haryadi is lecturer in ITB (Bandung Institute of Technology), Indonesia, since 1984. Currently teaches several courses: Traffic Engineering, Quality and Regulatory Services Telecommunications and Regulations. In addition to teaching at the ITB, Sigit Haryadi also becomes experts at the Directorate General of Post and Telecommunication Indonesia since 1986. Experience in the industry: since 1990 to become an expert in some Providers and Telecom Operators in Indonesia. Since 2001, Sigit Haryadi became associate professor in the institution.
\end{abstract}

\section{References}

[1] Sigit Haryadi. (2013). Telecommunication Traffic: Technical and Business Consideration. Lantip Safari Media. Bandung, Indonesia.

[2] Sigit Haryadi. (2013). Telecommunication Service and Experience Quality. Lantip Safari Media. Bandung, Indonesia.

[3] ETSI Standard TS 102 250-2 v2-2-1. (2011). Speech and multimedia Transmission Quality (STQ); QoS aspects for popular services in mobile networks; Part 2: Definition of Quality of Service parameters and their computation.

[4] ITU-T Recommendation G-1010. (2011). End-user multimedia QoS categories. 2011.

[5] GSM Association Standard PRD IR 42. (2001). Definition of quality of service parameters and their computation. 2001.

[6] ITU-T Recommendation E.800. (1984). Definitions of terms related to quality of service.

[7] ITU-T Recommendation G.1000 (1984). Communications Quality of Service: A framework and definitions.

[8] 3GPP Standard TS 23-207 V5-10-0. (2005). Technical Specification. 3rd Generation Partnership Project.

[9] ETSI Standard EG 202-057 V1-3-1. (2008). Speech Processing, Transmission and Quality Aspects (STQ). 
[10] 3GPP Standard TS 32-410. (2012). Telecommunication Management; Key Performance Indicators (KPI) for UMTS and GSM.

[11] 3GPP Standard TS 32.402. (2001). Telecommunication management; Performance Management (PM); Performance measurements. 2001.

[12] European Telecommunications Standards Institute, ETSI TS 102 250-1 V2-2-1. (2011-04). Speech and multimedia Transmission Quality (STQ), QoS aspects for popular services in mobile networks; Part 1. Assessment of Quality of Service.

[13] Haryadi, Sigit; Limampauw, Ivantius. (2012). QoS Measurement of Telephony Services In 3G Networks Using Aggregation Method. Conference Proceeding of TSSA 2012. Denpasar, Indonesia.

[14] Haryadi, Sigit; Nusantara, Sandy. (2012). QoS Measurement of Web Browsing Services In 3G Networks Using Aggregation Method. Conference Proceeding of TSSA 2012. Denpasar, Indonesia.

[15] Haryadi, Sigit; Pramudita, Arnold. (2012). QoS Measurement of Video Streaming Services in a 3G Networks Using Aggregation Method. Conference Proceeding of TSSA 2012. Denpasar, Indonesia.

[16] Haryadi, Sigit; Andina, Raisha. (2012). QoS Measurement of File Transfer Protocol Services In 3G Networks Using Aggregation Method. Denpasar, Indonesia. 2012.

[17] Hardy, C. William. (2001). QoS Measurement and Evaluation of Telecommunications Quality of Service. John Wiley \& Sons, Ltd. Baffins Lane, Chichester, West Sussex, PO19 1UD, England.

[18] ITU-T Recommendation G-107. (1984). The E-Model, a computational model for use in transmission planning. 